\title{
Insight Looks to Soft (Continuum) Robotics
}

\section{Sadique Shaikh* and Tanvir Begum}

KYDSC Trust's, Institute of Management \& Science (IMS), India

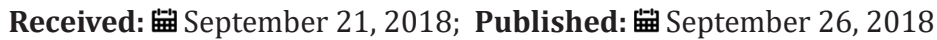

*Corresponding author: Sadique Shaikh, KYDSC Trust's, Institute of Management \& Science (IMS), Bhusawal, India

\section{Opinion}

Soft Robotics is emerging fresh sub field in Robotics which is very useful in medical, industry, space exploration, deep sea exploration, Nano-robotics and many more likewise applications. The major benefit of Soft Robots as compare to Rigid Robots their excellent flexibility and adaptability to accomplish task. Before to move further I would like to state Soft or Continuum Robots first "Soft Robots are small, medium and big shapes various biological or non-biological body forms robots which are made up using ultra soft and flexible materials, where materials are engineered using Continuum Mechanics and Kinematics". The big difference between conventional rigid robots and soft robots, in rigid robotics intelligence engineered using AI only to control robotics body, but in soft robotics the materials using which robots has made themselves smart and has intelligence, sensations and actuations. Therefore, Soft Robots can also learn from surrounding environment in self mode as well as has greater flexibility in clutching, climbing, moving, defending etc. why this happen? This would be question in your mind let me answer it why this happen. Because Soft Robotics constructed with highly compliant materials similar those originate and found in living organisms and creatures on planet earth. Hence Soft Robotics build up using material morphology and Continuum Mechanics, it's a mechanics that deal with the analysis of kinematics and mechanical behaviour of materials modeled as continuous mass rather than discrete particles, therefore Soft Robotics also called as "Continuum Robotics". These robots constructed using Biological materials, Biophotonics materials, Conductive polymers, Biochemical materials, Nanomaterials, Nanocomposites, Synthetic Biology, Shape Memory Alloy (SMA) and Smart Materials, DLC, Carbon having high young modulus and so on. In conclusion better to say Smart Materials are the building blocks of Soft/Continuum Robots, where smart materials can be defined as "Materials which has ability to sense some environmental stimuli, process and actuate (Response) according to sensation". Hence Soft Robots need less Electronic AI as compare to Rigid Robots and less harmful for human and environment as well as mimic and learn move and adapt quickly from surrounding (Figure 1). In above figure I have depicted some succeeded Soft Robots like Octobot world first ultrasoft and flexible Soft Robots, Soft Robot Fish etc.
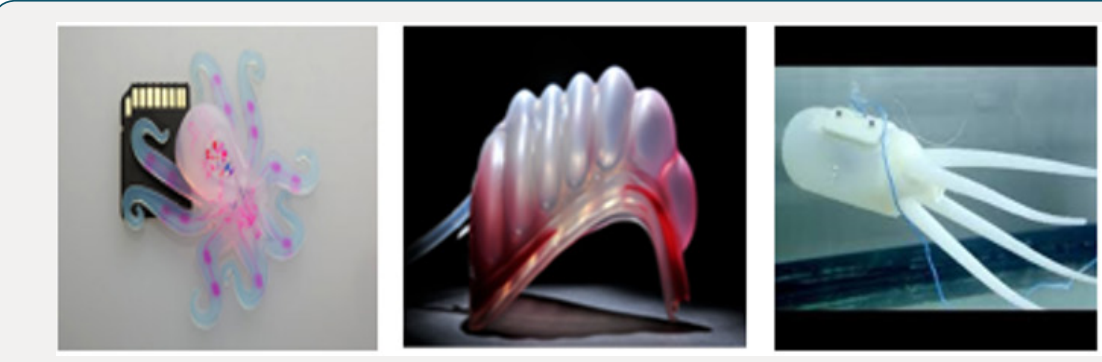

Figure 1.
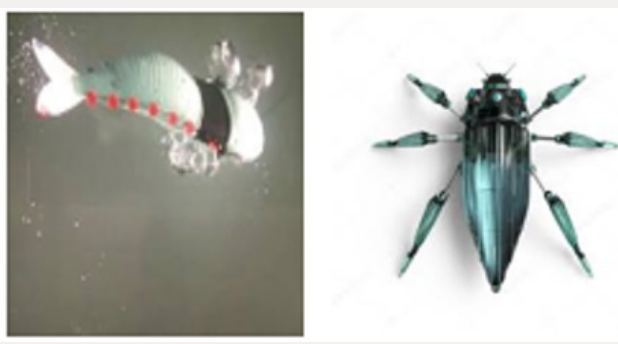

\section{Conclusion}

Soft or Continuum Robotic is fresh subfield in Robotics technology where lot of research need to do bring it on next level.
This branch of robotics has its own different important and utility along with conventional one and very useful in Deep Space, Medical, Industry and Deep Sea research. 
(C) (i) This work is licensed under Creative

To Submit Your Article Click Here:

DOI: 10.32474/ARME.2018.01.000106

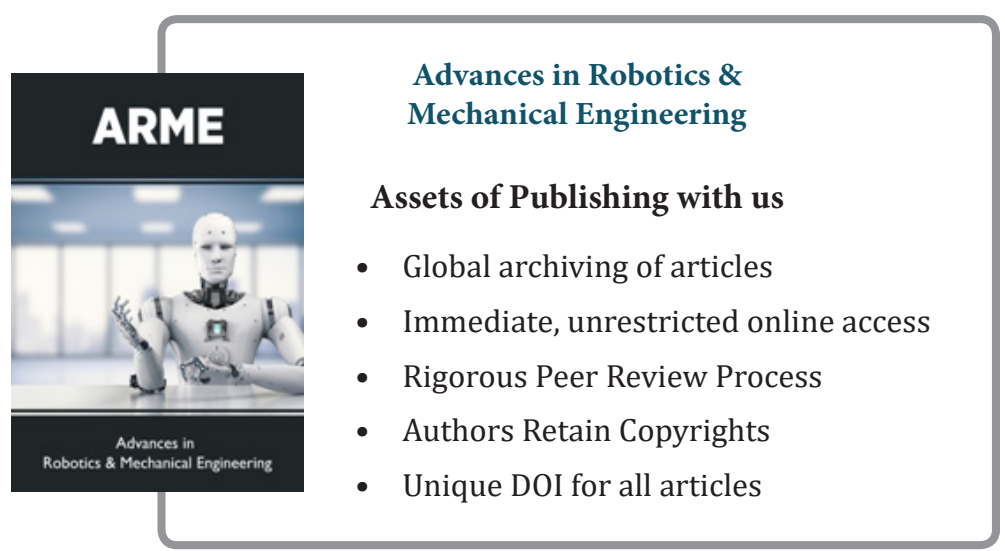

